\title{
Index
}

Abba Arsenius 106n.15

abbot 2-4, 10

Anselm of Bec see Anselm of Bec

authority of 85-7, 89-102

depositio drama see depositio drama

discipline 109n.52, 126, 195

'harming to help' see 'harming to help'

Lanfranc of Bec see Lanfranc of Bec

lordship 92

obedience 92, 89-102

as pastor $116-23$

political acumen 117-18, 129-34

second-generation reformers 61

vicar of Christ 92, 109n.45

William of Volpiano see William of Volpiano

Abelard, Peter 44, 146, 165-6, 187

Abraham and Issac 120-3, 140

Ademar of Chabannes 53

Aelred of Rievaulx 128, 138, 139, 141, $145,167,168,187$

affect vs. emotion $7-8$

affect theory $7-8$

affective devotion see affective piety affective piety

Anglo-Saxon 16n.47, 53

Anselmian 1-8

see also Anselm of Bec
Christus patiens see Passion of Christ

Cistercian 137, 154, 166-8

compassion 6, 93, 95-102, 110n.61, $163,168,176-7,191,194$

empathy 30, 43n.93, 50, 163, 168

female $18,31,42$ n.91, 45n.114, 118, 126-9

Franciscan 167, 168, 193

gift of tears 35 n. $5,52,59,700.33,170$

historiography of $1-8$

late medieval 154, 168-79

lay piety 133,190 on.134

Passion of Christ 29-32, 34, 48, 49-51,

$53,54,56,60,61,63,64,84,85$,

$87,91,93-7,104,105$ n. 5,125 ,

155-7, 160-79

Southern, Richard W. 6, 15n.36, $17 n .48,145 n .84,148 n .113$

affectivity see affective piety

affectus 26-9

Agnes of Poitou 19, 20, 22, 127-9

Ambrose, Aurelius 25, 39n.51, 85 . 105n.11, 106n.15, 112n.72, 139n.15

De fuga saeculi 85

De paradyso 106n.15

Anastasius the Venetian 72n.60, 155, 179n.3, 184 n. 51

De veritate corpore et sanguine domini 155 
Anselm of Bec 86, 148n.113, 150n.131 affective piety and 2-3, 6, 10, 18, $47,53,65$

John of Fécamp and 11n.9, 13n.23, 53 . 160-4

Prayers and Meditations 2, 20, 69n.16, $125,160-4,168-9,172,177$

Arians 95-102

arma Christi 176

Arne-Dannenberg, Paul 126

Assumption 132

Athanasius of Alexandria (Saint)

52, 97-102

Contra Arianos 97-102

Augustine of Hippo (Saint)

Confessions

Fécamp's copy of $66-7,85,103$

John's use of 23, 25, 39n.51, 4on.67, $42 n .84,47,56,65-7,68 n .10$, 81n.174, 82n.177, 111n.70, 112n.72 medieval reception of 52, 66-7, $126,154,162,165,172,174$

Contra Faustum 97-102

Contra Felicianum 100, 114n.91

De civitate dei 105n.12, 112n.72, 115n.94

Fécamp's copy of 85,112 n. 72 , 119-23, 140n.23

Manuale of Pseudo-Augustine 172-7

Fécamp's copy of 190 on.136

Meditations of Saint Augustine 1, 10, $172-7$

On the Correction of the Donatists 95

Retractiones 65, 81n.172

Baudril of Bourgueil 157, 164

Bec 66, 70n.36, 72n.6o, 86, 123, 126, 138n.7, $149 n .128,154,161,164,169,172,177$

blood relic $159,183 n .40$

Benedict of Aniane 46, 52

Benedict of Nursia 21, 101, 118, 162, 176

Benedictine reform see monastic reform

'Benedictines' 2, 4, 12n.11, 191, 192

'blind', communal ritual 2, 92
Berengar of Tours 19, 96, 112n.72, 118, $123-6,137,155,158,182 n .26$

Bernard of Clairvaux 4, 10, 65, 166-8, 172, $176,177,178,187 n .109,188$ n.120

Bestul, Thomas 137, 162

Bisson, Thomas 92, 109n.47

blood 1, 9, 29, 30, 43n.94, 49, 56, 61, $87,89,90,91,93,94,124$, $143 \mathrm{n} .59,172$

blood relic see relic of the Precious Blood of Christ

Bonaventure 176

De perfectione vitae ad sorores 176

Bonnes, Jean-Paul 19-20, 192

Brevis relatio (Fécamp chronicle) 131, 149n.123

Brionne 125

Caesarius of Arles 59, 76n.109

Caesarius of Heisterbach 176

Dialogus miraculorum 176

Cain and Abel 119, 139n.21

caritas 27-33, 40n.70, 41n.72, 42n.87, 83, 87, 89-96, 102, 104, 110n.61, 111n.67, 126, 155, 166-8, 177, 179, 187n.109, 192

Cassian, John 28, 41n.77, 104n.2, 106n.18 Cassiodorus, Flavius Magnus Aurelius $52,152 n .162$

Choy, Renie 49

Christ see Jesus Christ chronicles at Fécamp see Brevis relatio; Dudo of Saint-Quentin; Libellus de revelacione

Chrysostom, John 56, 59, 6o, 73n.73, $78 \mathrm{n} .123$

Cistercian piety 1, 10, 19, 35n.8, 36n.8, 137 , $154,166-8,187$ n.105, 188n.120, 193 carta caritatis 167

Cluny see also Maiolus of Cluny; Odilo of Cluny; Odo of Cluny influence on John of Fécamp 62-5 William of Volpiano and $62-5$ 
compassionate cruelty see 'harming to help'

compunction $51,52,56,59,64,85$, 106n.15, 162, 170

confessio $38 \mathrm{n} .33,82 \mathrm{n} .178,118$

Confessio fidei (John of Fécamp) 3, 19, 20, 21, 35n.5, 36n.9, 123-6, 142n.37, 143n. $58,155,169,188 \mathrm{n} .118$

authenticity of 36 n.9, 142n.37

Confessio theologica (John of Fécamp)

Byzantine sources 47, 56, 72n.69, 114 n. 89

Confessions of St Augustine and 10, $23,25,39 n .43,40 n .67,42 n .84,47$, 51, 52, 56, 65-7, 81, 82, 85, 103, 106, 122, 126, 128, 154, 164-8, 172, 174,192

male monastic audience for 19-24 manuscript at Bec 37n.19, 7on.36, 161, 174, 188n.118

medicine of penance 48,59

medieval circulation 11n.3, 36n.15-17, 37n.19, 125, 143n.58, 152n.162, 168-77, 188n.119-122

meditatio 52

performative 'I' 22

recensions of see Confessio fidei; Libellus de scripturis et verbis patrum collectus ad corum presertim utilitatem qui contemplativae vitae sunt amatores

wounding love see wounds see also compunction; contrition; confessio; conversio; 'dart of love'; emotional reform; Hannah; interiority; Jesus Christ; Mary Magdalene; palate of the heart; tears

Confessions see Augustine of Hippo Constable, Giles 137 contrition 23, 38n.27, 43n.92, 51, 52, 6o, $61,63,64,65,67,85,87,107$ n.25, $118,136,159,163,170$ conventio 92

conversio 18, 24, 38n.33, 66, 82n.178, 118, $133,135,137,157,162,164,165$

Courcelle, Pierre 65

Crespin, Gilbert 164

cross see also depositio drama; Jesus Christ

Adoration of the Cross 49

devotion to 29-31, 83-5

Exaltation of the Cross 56, 81n.167

Invention of the Cross 56

Cuxa, Saint-Michel 37n.19

Damian, Peter 55-6, 71n.56

'dart of love' 30, 96, 102, 115n.95, 177

Davis, Robert Glenn 7

Deleuze, Gilles 7

depositio drama 86-9, 91, 106n.19, 109n.45, 160, 164, 183n.42

devotio moderna 174

discipline in the monastery $52,63,84$,

$$
\text { 89-102, } 195
$$

Dol 157, 164

Donatists 95-7, 111n.68, 112n.72

Dudo of Saint-Quentin 133, 147n.105, 150n.140-1

Durandus of Troarn 96, 112n.72, 123, 125, 141n.36, 155

Easter drama see visitatio sepulchri

Easter sepulchre 61, 86-9, 106n.19, 159, 16o, $183 n .42$

Easter Sunday 8on.152, 86-9

emotion as an invention of the nineteenth century 17 n. 55

emotional reform 4, 9, 18, 23, 24, 29, 34, $46,54,58,83,89,111 \mathrm{n} .70,125$, $137,192,193$

Ephraem the Syrian 56, 59, 61, 67, 75n.94, 77n.118, 78n.120, 85, 119, 126, 172

De compunctione cordis 6o, $76 \mathrm{n} .105$, 169-70, 188n.122

eremitic spirituality see hermits 
Eucharist 19, 23, 35n.7, 62, 64, 96, 113n. $78,118,120,123,124,125$, $131,133,142 \mathrm{n} .44,155,158,182 n .27$ heresy of Berengar of Tours see Berengar of Tours

Evreux 122, 137, 179n.4

Faustus the Manichean 97-102, 115n.94 Fécamp abbey archaeology 4, 13n.22, 181n.22 architecture xix, 4, 13n.22, 130, 146-7n.105

blood relic 4, 143n.59, 149n.124, 156, 157-6o, 177, 181-2n.19-30 charters 3, 4, 57, 132, 147n.110, 149n.125-7

chronicles see Brevis relatio; Libellus de revelacione

donations to the abbey 129-34

ducal palace and xix, 130 economic holdings 12n.24, 129-34, 138 n.9

education at the monastery $47-54$ etymology of 158

exemption for 4, 12n.18, 129, 134, 147n.111, 151n.151, 181n.21

Norman conquest and 4, 12n.15, 149n.122

Norman dukes and 3, 9, 53, 56, 57 , 130, 129-34, 146n.105, 147n.108, 149n.122, 151n.151, 157, 181n.23

pilgrimage to $122,157,181 \mathrm{n} .22$ relic to Mary Magdalene 33, 44n.108, $87,108 \mathrm{n} .31$

school for priests $58,122,132$, $149 n .128$

Franciscan piety $1,167,168,193$

Fruttuaria 61, 75n.89, 79n.131, 81n.161, 105n.7, 106n.19, 107n.26, 108n.33

Fulton, Rachel 31, 137

Gelduin of Mont Saint-Michel 100 Geoffrey of Vendôme 92
Gerbert of Saint Wandrille 9, 159, 163 , $177,180 n .8$

Scriptura 154-7, 180n.8, 182n.27, $183 n .33$

Gerson, Jean 174

Glaber, Rodulfus 58, 74n.79, 75n.88, 122, 131, 133, 140n.27, 148n.116, 150n.135, 183 n. 4 O

Glastonbury 125, 143n.59

blood relic 159, 182n.25, $183 n .34$

Good Friday drama see depositio drama

Gorze 2, 6o, 77n.120, 106n.19, 107n.26, $138 \mathrm{n} .9,150 \mathrm{n} .134$

Goscelin of Saint Bertin 67, 128, $145 \mathrm{n} .87,165,186 \mathrm{n} .88$

grace $8,24,28,32,52,53,54,93,124,159$, 165,176

Gregorian reform see monastic reform

Gregory of Nazianzus 56

Gregory the Great 9, 124

Moralia in Job 47, 51-2, 63, 7on.27, $82 n .178,116,132$

Pastoral Care 116-23

Guibert of Nogent 154, 177, 184n.49, 193

Monodies 16o-6, 186n.89-96

Guitmund of Aversa 123

habitus 5, 86

Hallinger, Kassius 64

Hamburger, Jeffrey 174, 176, 177

Hannah 8, 24, 29, 31-4, 43, 44, 45, 47, $56,68,73 n .76,83,85,90,102$, 105n.12, 108n.38, 172, 179, 192

'harming to help' 89-102, 110n.61, 177

Henry of Sully 159

Herbert Losinga 159, 182n.30

heresy see Arians; Donatists

Herluin of Bec 72n.6o, 164, 186n.84

hermits $12 n .14,47,55,56,59,71 \mathrm{n} .54$,

72n.59-62, 78n.124, 89, 91,

$93,94,95,96,102,111,122$,

$127,130,132,155,167,180 n \cdot 5$,

188 n.124

Hildebert of Lavardin 92 
Hilgarth, Jocelyn 119

history of emotions $7-8,10,14 \mathrm{n} .27$,

15n.35, 16n.44-47, 17n.50-56,

18, 26-9, 38n.26, 41n.78, 161,

164,169

Hollywood, Amy 23, 93

homilies at Fécamp 9, 42n.91, 56, 59,

6o, 61, 62, 63, 73n.75, 76n.109,

77n.114, 78n.123, 79n.141,

8on.152, 81n.159, 84-5, 86, 88,

89, 92, 102, 104n.1, 108n.32,

112n.72, 113n.8o, 140n.122,

$145 \mathrm{n} .85,176$

Hugh of Saint-Victor 172

individual 2, 6, 22-5, 55, 59, 64, 132, 137 , 166, 174, 186n.102

'discovery of the individual'

historiography 5-6, 15n.36-16n.39, 150n.133, 162, 194-6

interiority 18, 24-6, 29, 31, 32, 47, 52, 53, $54,55,56,59,60,63,64,67,83$, 89, 91, 102, 104, 109n.41, 111n.7o, $118,122,124,129,137,159,163$, 192, 193, 194, 195

Iogna-Prat, Dominique 133

Isidore of Seville 52, 59, 75n.94, 76n.98, 104n.3

Ivo of Chartres 164

Jerome (Saint), Eusebius Sophronius $24,112 n .72$

Jerusalem, pilgrimage to 53,132

Jesus Christ see also affective piety; depositio drama

Christus Pastor 119, 120, 122

crucified, suffering 1, 2, 6, 7, 8, 9, 10, 27, 29-31, 48, 49, 50, 51, 53 ,

$54,56,60,64,68,84,85,91$,

92, 94, 102, 104, 117, 155, 156,

$157,159,160,163,166-8,169$,

172, 176, 177

deposition see depositio drama empathy 50, 64, 163, 168

humanity 34, 94, 102, 104,

193, 194n.72

Job see Gregory the Great

John of Reims 155

John of Salerno 63

John the Evangelist 101, 170

jongleurs at Fécamp 157, 158, 181n.18

Joseph of Arimathea 158, 160, $183 n .33$

Jumièges 66, 8on.151, 82n.181, 148n.119, 165,186 n. 87

La Chaise-Dieu 162

Lament for Lost Solitude (John of Fécamp) 35, 8on.7, 134-6, $137,1521.162$

Lanfranc of Bec 47, 56, 72n.58, 86, 106n.16, 123, 125, 141n.35, 143n.54, 149n.128, 151n.155, 161

Lazarus 63

Leclercq, Jean 19, 20, 64, 137, 192

Lecouteux, Stéphane 59

lectio divina 5, 35, 38n.25, 41n.77, 51, 69n.14, 168, 179

Lent 85, 103

Letter, Ad monachos dyscolos (John of Fécamp) 89-93

Letter, Tuae Quidem (John of Fécamp) 126-9

Letter to the abbot of Saint-Berthe of Blagny (John of Fécamp) 134

Letter to the anonymous nun (John of Fécamp) 19, 112n.76, 126-9, 189 n. 128

Letter to the bishop William of Evreux

(John of Fécamp) 122, 137,

179n.4

Letter to the Empress Agnes (John of Fécamp) 19, 20, 22, 126-9, 135, $144 \mathrm{n} .79,183 \mathrm{n} .4 \mathrm{O}$

letters in the Middle Ages 36 n.15, 37n.20, 144n.68 
Leo IX (Pope) 123, 182

John of Fécamp as papal legate 3, 122 Letters between Leo IX and John $134,141 \mathrm{n} .26$

libelli precum 46, 47, 48-5o, 69n.15, 700.23

Libellus de revelacione 57, 74n.86, 75n.88, $151 \mathrm{n} .151,181 \mathrm{n} .21$

Libellus de scripturis et verbis patrum collectus ad eorum presertim utilitatem qui contemplativae vitae sunt amatores (John of Fécamp) 3, 19, 21, 22, 35n.5, 36n.15, 37n.22, 42n.9o, 43n.96, 44n.109, 110n.56, $123,145 \mathrm{n} .85,169,188 \mathrm{n} .118$

liturgical customs at Fécamp comparable liturgies 86,87 , 106n.19, 106n.21

liturgical manuscripts 107n.26 William of Volpiano and 57, 61, 193 see also Assumption; depositio drama;

Easter sepulchre; Nativity;

Purification of the Virgin; Quem quaeritis; visitatio sepulchri

longing for the divine 9, 13n.23, 21, 29,

32, 44n.104, 47, 48, 52, 6o, 65,

$68,73 n .78,112 n .76,117,129,135$,

$145^{n} .88,151 \mathrm{n} .155,177,185$ n.6o, 194

Lucca blood relic 159, 181n.24, 183n.33

Ludolfus of Saxony 2

Lyre 66, 82n.183

Maiolus of Cluny 55, 57, 62, 64, 74n.79, 75n.88, 79n.139, 81n.162, 127, 144

Manicheans 96, 98, 115n.94

Manuale of Pseudo-Augustine see Augustine of Hippo manuscripts from Fécamp's library

Barcelona, Ripoll ms. 214 viii, 36n.17, 37 n.19, 170

BnF ms. lat. 1684 viii, 97, 106n.15

BnF ms. lat. 1714 58, 62, 63, 75n.94, 76n.106, 77n.118, 78n.120, 105n.13
BnF ms. lat. 1919 19, 35n.3, 36n.17, 152n.162, 187n.105, 188n.120

BnF ms. lat. 2055 105n.12, 112n.72, 119, 140n.22

BnF ms. lat. 2079 73n.76, 97, 99, 100, $112 n .72$

BnF ms. lat 3088 19, 21, 35n.7, 37n.19, $144 \mathrm{n} .68,152 \mathrm{n} .162,188 \mathrm{n} .118$

BnF ms. lat 5290 62, 79n.145

BnF ms. lat. 13593, 36n.16, 37n.19, 7on.36, 145n.85, 174, 188n.118, $189 n .130$

Metz, Mediathèque ms. 245, 36n.16, 37n.19, 61, 69n.16, 108n.31, 142n.45, 174, 188n.118, 189n.127

Montpellier, Bibliothèque interuniversitaire, ms. $\mathrm{H}_{309}$ 36n.16, 37n.19, 125, 188n.118

paleography of 4

Rouen, BM ms. 140062

Rouen, BM ms. 1404 62, 79n.141, $81 n .159$

Vienna, Österreichische Nationalbibliothek, Cod. 1580 36n.17, 169, 170n.105, 188n.121

Vienna, Österreichische Nationalbibliothek, Cod. 1582 36n.17, 169, 170, 188n.121

Zwettl, Stiftsbibliothek, Cod. 164, viii, 36n.17, 144n.68, 145n.82,

Martha 32 17o, 172, 189 n.128

Mary see Virgin Mary

Mary Magdalene 163, 172, 179, 192

Confessio theologica 8, 24, 29, 31-4, 41n.74, 43n.106-45n.114, 56, 61-5, 68, 8on.148-156, 81n.159-161, 83, $87,89,102,108$ n.134, 155, 163, $172,179,192$

Mary of Egypt 59, 6o, 63, 77n.114, 78n.125 Marys, at the tomb 87-9, 108n.31

Massumi, Brian 7, 17n.52

Matilda of Tuscany 161 
Maundy Thursday 89

Maurilius of Rouen 9, 122, 125, 137, 159, 161, 179, 18on.4, 184n.59, 189n.129

Oratio ad sancta Mariam 155

McGinn, Bernard 137

McNamer, Sarah 11n.6, 17n.50, 36n.15, $38 n .25,145 n .83-4$

Meditations of Saint Augustine see Augustine of Hippo

Melchisedech 115n.94, 119, 120, 121, 122,140 on.22

mendicants see Franciscan piety

monachos dyscolos see letter, Ad monachos dyscolos

monastic discipline see discipline in the monastery

monastic reform see also Cistercian piety

'crisis of coenobitism' 4, 14n.29, 71, 139n.12

customs and rules 2, 3, 10, 46, 47, 55, $5^{8-65}, 67,75^{\text {n.95, }}$ 79n.129, 86, 106n.19, 107n.26, 118, 126, 132, 149n.124, 181n.22, 182n.30

emotional reform 4, 9, 18, 22-9, 32, $33,34,54,56,83,89,91,102,103$, 111n.70, 192-5

historiography of 14n.25-6, 15n.32, $136-8$

Mont Saint-Michel 71n.54, 72n.6o, 73n.7o, 100, 107n.26, 114n.89, 119, 139n.20, 143n.65, 155, 179n.3

Montivilliers 128

Morrison, Karl 93

Nativity 132

Nicodemus 156, 158, 160, 182n.27, $183 n \cdot 33,184$ n. 45

Nogent-sous-Coucy 161

Norman dukes 9, 57, 131, 132, 133, 146n.105, 147n.108, 149n.149, 158

Norwich 107n.21, 159, 182n.30, 183n.32

Notre-Dame-aux-Nonnais 128

novices $46,55,62,84-5,92,104$ n.1-2, 120 obedience in the monastery $55,58,62$, 64, 89-95, 102, 103, 109n.52, 110n.52, 134, 140n.22, 145n.88, 151n.148, 170, 194, 196n.6

Odilo of Cluny 53, 62, 63, 64, 74n.85, $79 n .139,81 n .163,107 n .21$

Odo of Cluny 62-5, 67, 79n.139,

8on.146, 122

Occupatio 63, 79 n.144

Ordericus Vitalis 141n.36, 179, 183n.33

palate of the heart 23-6, 39n.53,

$$
112 n .76,129
$$

passiones 8

pastoral care 116-23, 126, 127, 128, 129, 130, 136, 138n.2, 140n.28, 141n.28, 146n.95, 149n.126, 152n.161, 195

Paul of Saint Père de Chartres 100 penance $5,48,49,51,53,59,60,61,63$, $64,81 \mathrm{n} .164,85,127,132$

Peter of Blois 64, 81n.165

Peter of Jumièges 165

Peter the Venerable 133

Phipps, Colin 55

Pilate, Pontius 16o, 184n.45

Précieux-Sang, relic of see relic of the Precious Blood of Christ

Premonstratensian 19, 188n.120 prior $3,11 n .8,18,19,20,35$ n. 3, 46, 55, $57,59,62,67,86,115$ n.99, 159, 161,165

psalms xv, 23, 41, 46, 47-50, 57, 68n.10, $69,114 n .89,128$

Purification of the Virgin 132

Quem quaeritis 79n.129, 87, 89, 107n.26

Ravenna 9, 23, 46, 47, 51, 54-7, 65, 67, 68,71 1n.54, 96, 120-2, 140n.25, $188 \mathrm{n} .124,192$

Reddy, William 7, 17, $38 \mathrm{n} .26$ reform, monastic see monastic reform 
relic of the Precious Blood of Christ 143n.59, 149n.124, 156-6o, 177, 181n.20-184n. 46

Renaissance humanism 194

Richard I of Normandy (Duke) 130-4, 146n.105, 149n.124, 157, 158, $181 n .22$

Richard II of Normandy (Duke) 3 , $53,56,57,58,130-4,147$ n.108, 149n.124, 151n.151

Richard of Saint Vanne 53, 54

Richard of Saint-Victor 24

Ripoll 37n.19, 170, 188n.124

Robert of Saint-Quentin 164

Robert of Tomblaine 161, 184n.47

Robert of Torigny 92

Robertson, Duncan 137

Romuald of Ravenna 55-7, 60, 67, $71 \mathrm{n} .54,72 n .57,76 \mathrm{n} .102,122$, 188n.124

Rosenwein, Barbara 8, 14n.27, 15n.33, $17 n .56,38 n .29,40 n .69$

Rothschild Canticles 115n.95, 174-7, 190n. $137-50$

Rouen 9, 56, 134, 137, 155, 159

Rubenstein, Jay 165, 186n.89-90

Rule of Saint Basil 59, 60, 76n.107, $77 \mathrm{n} .12 \mathrm{O}, 78 \mathrm{n} .124$

Rule of Saint Benedict (RB) 20-1, 23, 28, 41n.76, 42n.92, 46, 55, 57, $58,62,75$ n.88, 90-3, 95-6, 102, 103, 105n.6, 109n.45, 110n.61, 115n.100, 122, 134, 137, 139n.11, 141n. 31,149 n.129

Saenger, Paul 52

Saint-Arnoul in Metz 37n.37, 57, 6o, 61, 77n.120, 79n.131, $143 n .65,169$

Saint-Bénigne de Dijon 3, 9, 37n.19, 46, $51,54,55,59,61,62,67,71 \mathrm{n} .53$, 74n.79, 75n.89, 79n.127, 8on.146, 106n.19, 111n.63, 115n.99, 125,
126, 130, 143n. 57,144 n.79,

148n.116, 169, 187n.105, 192

Saint-Berthe of Blagny 134, 144n.66

Saint-Etienne in Caen 86

Saint-Evroult 66, 72n.6o, 155

Saint-Germer-de-Fly 67, 161

Saint-Ouen 66, 82n.182, $107 n .26$

Saint-Wandrille 9, 13n.19, 72n.6o, 100, 114 n. $85,154,155,159,163,177$, 18on.8, 182n.27, 183n.33

Sant'Apollinare in Clase viii, 54, 55, 56, 73n.72, 120, 121, 130

see also novices; Romualdus of Ravenna Scheer, Monique 15n.35, 41 n.78 self-awareness see individual sermons see homilies at Fécamp Shenoute 92, 109n.49

Sinai 56

Smaragdus of Saint-Mihiel 46, 52, 75n.94, 76n.99, 170, 172, 188n.122, 189 n.127

Diadema monastica 58, 59, 6o, 68n.2, 75n.94, 76n.99, 77n.119, 85, 169

Expositio in regulam sancti Benedicti 75n.94, 76n.98

Song of Songs 3o, 41n.72, 42n.91, 46, 161, $174,176,190$

see also 'dart of love'

Southern, Richard W. see affective piety students of John of Fécamp see Anselm of Bec; Durandus of Troarn; Gerbert of Saint-Wandrille; Herbert Losinga; Maurilius of Rouen; Robert of Tomblaine

suffering see affective piety Summe sacerdos (John of Fécamp) 142n.45 Symeon the New Theologian $5^{6}$

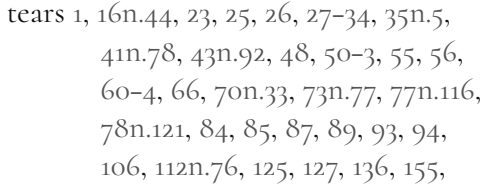


Index

159, 162, 169, 170, 172, 18on.13,

183n.40, 190, 191

see also affective piety

Theodore of Mopsuestia 59

Third Lateran Council 167

Thomas à Kempis 2, 174

tonsure $89,98,99,101,170,171$

Troyes 19, 36n.17, 128, 143n.58, 187n.105, $188 n .120$

Tuae Quidem letter see Letter, Tuae Quidem

Turin 96

twelfth-century Renaissance

$$
\text { 15n.36, } 194
$$

\section{Vanderputten, Steven 61}

Vigilius Tapensis 97, 113n.81

De Trinitate 97

Virgin Mary 31, 49, 50, 64, 73n.69, 183n.34, 189n.129

visitatio sepulchri 79n.129, 107n.26, 108n.35, 130

Vulfran of Saint-Wandrille 10o, $114 n .85$

vulnera see wounds
Warin of Metz 96-7, 101, 113n.78,

$$
126,167
$$

weeping see tears

White Monastery, Egypt 92

William of Volpiano 3, 4, 7, 9, 12n.13, 21, 37n.19, 46, 47, 55, 57-62, 63, 67, 71n.54, 74n.79, 75n.88, 76n.97, 78n.128, 79n.127, 81n.161, 86, 96, 105n.7, 106n.19, 107n.26, 111n.63, 115n.97, 122, 126, 140n.27, 144n.72, 15on.134, 161, 170, 181n.23, 184n.47, 188n.124, $189 n .124$

William the Conquerer 2, 12n.15, 103, 125, 134, 137, 146n.105, 149n.122, 1511.149

Ship List 2, 149 n.122

Wilmart, André 2, 19, 20, 191 wounding see wounds

wounds 1, 18, 27, 30, 31, 39n.46, 41n.72, 42n.91, 43n.92, 48, 49, 50, 6o, 69n.11, 7on.28, 78n.121, 83, 84, $93,94,102,125,159,167,172$, 175-7, 189n.125, 190n.143, 192 ostentatio vulnerorum 176 